\title{
On the minimization of Dirichlet eigenvalues
}

\author{
M. van den Berg * \\ School of Mathematics, University of Bristol \\ University Walk, Bristol BS8 1TW \\ United Kingdom \\ mamvdb@bristol.ac.uk
}

14 October 2014

\begin{abstract}
Results are obtained for two minimization problems:

$I_{k}(c)=\inf \left\{\lambda_{k}(\Omega): \Omega\right.$ open, convex in $\left.\mathbb{R}^{m}, \mathcal{T}(\Omega)=c\right\}$,

and

$$
J_{k}(c)=\inf \left\{\lambda_{k}(\Omega): \Omega \text { quasi-open in } \mathbb{R}^{m},|\Omega| \leq 1, \mathcal{P}(\Omega) \leq c\right\},
$$

where $c>0, \lambda_{k}(\Omega)$ is the $k^{\prime}$ th eigenvalue of the Dirichlet Laplacian acting in $L^{2}(\Omega),|\Omega|$ denotes the Lebesgue measure of $\Omega, \mathcal{P}(\Omega)$ denotes the perimeter of $\Omega$, and where $\mathcal{T}$ is in a suitable collection of functions. The latter includes the perimeter of $\Omega$ and the moment of inertia of $\Omega$ with respect to its centre of mass.
\end{abstract}

Keywords: Dirichlet eigenvalues; Convexity constraint; Perimeter; Lebesgue measure; Moment of inertia

2010 Mathematics Subject Classification: 49Q10, 49R05, 35J25, 35P15

${ }^{*}$ Partially supported by the London Mathematical Society, Grant 41217 and by The Leverhulme Trust, International Network Grant Laplacians, Random Walks, Bose Gas, Quantum Spin Systems. The author wishes to thank Brian Davies and Dorin Bucur for helpful discussions. 


\section{Introduction}

Let $\Omega$ be an open set in Euclidean space $\mathbb{R}^{m}(m=2,3, \cdots)$, with boundary $\partial \Omega$, and let $-\Delta_{\Omega}$ be the Dirichlet Laplacian acting in $L^{2}(\Omega)$. It is well known that if $\Omega$ has finite Lebesgue measure $|\Omega|$ then $-\Delta_{\Omega}$ has compact resolvent, and the spectrum of $-\Delta_{\Omega}$ is discrete and consists of eigenvalues $\lambda_{1}(\Omega) \leq \lambda_{2}(\Omega) \leq \ldots$ with $\lambda_{j}(\Omega) \rightarrow \infty$ as $j \rightarrow \infty$. The Faber-Krahn inequality (Theorem 3.2.1 in [9]) asserts that if $c>0$ then

$$
\inf \left\{\lambda_{1}(\Omega): \Omega \text { open in } \mathbb{R}^{m},|\Omega|=c\right\}
$$

is attained for a ball with Lebesgue measure $c$. The Krahn-Szegö inequality (Theorem 4.1.1 in [9]) asserts that if $c>0$ then

$$
\inf \left\{\lambda_{2}(\Omega): \Omega \text { open in } \mathbb{R}^{m},|\Omega|=c\right\}
$$

is attained for two disjoint balls each with Lebesgue measure $c / 2$. For higher Dirichlet eigenvalues $(k>2)$ it is not known whether the variational problem

$$
\inf \left\{\lambda_{k}(\Omega): \Omega \text { open in } \mathbb{R}^{m},|\Omega|=c\right\}
$$

has a minimizer. However, it has been shown that if the collection of open sets in (11) is enlarged to the quasi-open sets then the variational problem

$$
M_{k}(c)=\inf \left\{\lambda_{k}(\Omega): \Omega \text { quasi-open in } \mathbb{R}^{m},|\Omega|=c\right\}
$$

has a bounded minimizer 2, 15, with finite perimeter 2. Even though the class of quasi-open sets with measure $c$ is much larger than the class of open sets with measure $c$, the infima under (1) and (2) are equal.

Few facts are known about these minimizers. E. Oudet has shown that the ball is not a minimizer of (10) for $k=3, m=3$. Furthermore the disc is a local minimum of the functional under (11) for $k=3, m=2$ [9], and any minimizer of (11) or (2) for $m=2, k=3, m=3, k=3$, and $m=3, k=4$ is connected [18, 1]. An upper bound for the number of components of a minimizer of (11) (or (22) has been obtained in Theorems 1 and 2 of 1 in terms of $k$ and $m$.

Minimization problems for Dirichlet eigenvalues with other constraints such as torsional rigidity or perimeter have been investigated in [12, 13] and 4, 7] respectively. In [7] it was shown that if $m=2,3, \cdots, k \in \mathbb{N}$, and if $\mathcal{P}(\Omega)$ denotes the perimeter of $\Omega$ then

$$
P_{k}(c)=\inf \left\{\lambda_{k}(\Omega): \Omega \text { open in } \mathbb{R}^{m}, \mathcal{P}(\Omega)=c,|\Omega|<\infty\right\}
$$

has a minimizer with a regular boundary, and that any minimizer is connected. The situation is very simple for $m=2$ : taking the convex envelope of a component of a planar open set decreases both its perimeter and all of its Dirichlet eigenvalues. It follows that if $m=2$ then any minimizer is convex and has diameter bounded by $c / 2$. See, for example, Theorem 4 in [1]. Further progress was made by Bucur and Freitas [5] who proved that if $m=2$, and if $\left(\Omega_{k}^{*}\right)_{k \in \mathbb{N}}$ is a sequence of minimizers of (3) for $k \in \mathbb{N}$ respectively then there exists a sequence of translates of these minimizers again denoted by $\left(\Omega_{k}^{*}\right)_{k \in \mathbb{N}}$ such that $\Omega_{k}^{*} \rightarrow \frac{c}{2 \pi} D$ as $k \rightarrow \infty$, where $\frac{c}{2 \pi} D$ is a homothety of a disc $D$ with radius 1 by a factor $\frac{c}{2 \pi}$. The convergence is with respect to the Hausdorff metric. As 
these authors point out in 5 it is not known whether the minimizers of (3) are convex for $m>2$ or whether their diameters are bounded uniformly and independently of $k$, see [2, 15].

In this paper we consider a class of constraints, which includes perimeter and moment of inertia, under the additional constraint of convexity. Let

$$
I_{k}(c)=\inf \left\{\lambda_{k}(\Omega): \Omega \text { open, convex in } \mathbb{R}^{m}, \mathcal{T}(\Omega)=c\right\},
$$

where $\mathcal{T}$ satisfies the following hypotheses.

(a) $\mathcal{T}$ is a set function defined on the open, convex sets in $\mathbb{R}^{m}$ which is (i) invariant under isometries, (ii) monotone, i.e. $\Omega_{1}, \Omega_{2}$ convex with $\Omega_{1} \subset \Omega_{2}$ implies $\mathcal{T}\left(\Omega_{1}\right) \leq \mathcal{T}\left(\Omega_{2}\right)$, (iii) non-negative and $\mathcal{T}(\Omega)=0$ if and only if $\Omega=\emptyset$.

(b) There exists $\tau>0$ such that if $\alpha>0$, and if $\Omega$ is open and convex then $\mathcal{T}(\alpha \Omega)=\alpha^{\tau} \mathcal{T}(\Omega)$.

(c1) $T^{*}$ defined by

$$
T^{*}=\inf \left\{\mathcal{T}(\Omega): \Omega \text { open, convex in } \mathbb{R}^{m},|\Omega|=1\right\}
$$

is strictly positive.

(c2) There exists an open, convex set $D$ with $|D|=1$ which is unique up to isometries such that

$$
\mathcal{T}(D)=T^{*}
$$

(d) There exist constants $K<\infty$ and $t>1 / \tau$ such that if $\Omega$ is open, bounded and convex then

$$
\operatorname{diam}(\Omega) \leq K \mathcal{T}(\Omega)^{t}|\Omega|^{(1-t \tau) / m} .
$$

We remark that (a) and (c2) imply (c1). Our first result is the following.

Theorem 1. Let $m=2,3, \cdots$ and let $k=1,2, \cdots$.

(i) If $\mathcal{T}$ satisfies (a), (b) and (c1) then variational problem (4) has a minimizer.

(ii) If $\mathcal{T}$ satisfies (a), (b), (c2) and (d), and if $\left(\Omega_{k}^{*}\right)_{k \in \mathbb{N}}$ are minimizers of (4) for $k \in \mathbb{N}$ respectively then there exists a sequence of isometries of these minimizers again denoted by $\left(\Omega_{k}^{*}\right)_{k \in \mathbb{N}}$ such that

$$
\Omega_{k}^{*} \rightarrow\left(\frac{c}{\mathcal{T}(D)}\right)^{1 / \tau} D
$$

where the convergence is with respect to both the Hausdorff metric and the complementary Hausdorff metric.

In [10] the authors study variational problem (44) in the case where $\mathcal{T}$ is Lebesgue measure, and obtain properties of minimizers. Here we note that the Lebesgue measure constraint satisfies (a), (b) and (c1). Theorem 1(i) confirms the existence of a minimizer in that case. However, this constraint does not 
satisfy (c2) nor does it satisfy (d). So we do not obtain any information about the asymptotic behaviour of these minimizers for large $k$.

We remark that if $\mathcal{T}_{1}$ and $\mathcal{T}_{2}$ are constraints which satisfy (a), (b) and (d) with constants $\tau_{1}, t_{1}, K_{1}$ and $\tau_{2}, t_{2}, K_{2}$ respectively and if there exists a convex set $D$ such that (c2) holds for both $\mathcal{T}_{1}$ and $\mathcal{T}_{2}$ then $\mathcal{T}_{1} \mathcal{T}_{2}$ defined by $\left(\mathcal{T}_{1} \mathcal{T}_{2}\right) \Omega=$ $\mathcal{T}_{1}(\Omega) \mathcal{T}_{2}(\Omega)$ satisfies (a), (b) with $\tau=\tau_{1}+\tau_{2}$, (d) with $t=\frac{t_{1} t_{2}}{t_{1}+t_{2}}$ and $K=$ $\max \left\{K_{1}, K_{2}\right\}$, and (c2) with $D$.

We defer the proof of Theorem 1 to Section 2. There we also present and prove some of its corollaries.

Our second result is an interpolation between the minimization of the $k^{\prime}$ th eigenvalue with a Lebesgue measure constraint, and of the $k^{\prime}$ th eigenvalue with a perimeter constraint. Since existence of a minimizer of the former has been shown for quasi-open sets, we define

$$
J_{k}(c)=\inf \left\{\lambda_{k}(\Omega): \Omega \text { quasi-open in } \mathbb{R}^{m},|\Omega| \leq 1, \mathcal{P}(\Omega) \leq c\right\} .
$$

We denote by $\mathfrak{M}_{k}$ the collection of minimizers of $M_{k}(1)$, and by $\mathfrak{P}_{k}$ the collection of minimizers of $P_{k}(1)$ respectively. These collections are non-empty by the results of [2, 15] and [7] respectively. Let

$$
\pi_{k}=\inf \left\{|\Omega|: \Omega \in \mathfrak{P}_{k}\right\},
$$

and

$$
\mu_{k}=\inf \left\{\mathcal{P}(\Omega): \Omega \in \mathfrak{M}_{k}\right\} .
$$

We also denote by $\omega_{m}$ the Lebesgue measure of the ball in $\mathbb{R}^{m}$ with radius 1 .

Theorem 2. Let $m=2,3, \cdots$, and let $k=1,2, \cdots$.

(i) $c \mapsto J_{k}(c)$ is monotonically decreasing, continuous on $\mathbb{R}^{+}$, and

$$
c_{1}^{2 /(m-1)} J_{k}\left(c_{1}\right) \leq c_{2}^{2 /(m-1)} J_{k}\left(c_{2}\right), \quad 0<c_{1} \leq c_{2}<\infty .
$$

(ii) If $c>\mu_{k}$ then there exists $\Omega^{*} \in \mathfrak{M}_{k}$ which is a minimizer of (9). If $c \geq \mu_{k}$ then

$$
J_{k}(c)=M_{k}(1) .
$$

(iii) If $0<c<\pi_{k}^{-(m-1) / m}$ then there exists $\Omega_{*} \in \mathfrak{P}_{k}$ such that $c^{1 /(m-1)} \Omega_{*}$ is a minimizer of (91). If $0<c \leq \pi_{k}^{-(m-1) / m}$ then

$$
J_{k}(c)=c^{-2 /(m-1)} P_{k}(1) .
$$

(iv)

$$
\mu_{k} \geq m \omega_{m}^{1 / m}
$$

$$
\pi_{k} \leq m^{-m /(m-1)} \omega_{m}^{-1 /(m-1)}
$$

$$
\pi_{k} \geq(2 m)^{-m /(m-1)}(m+2)^{-m / 2} \omega_{m}^{-1} .
$$

We do not have a proof of existence of a minimizer of (9) for $\pi_{k}^{-(m-1) / m}<$ $c \leq \mu_{k}$. However, the proof of Theorem 2 does not rely on that existence. We defer the proof of Theorem 2 to Section 3 . 


\section{Proof of Theorem 1}

Throughout we will denote the inradius of a set $A$ by

$$
\rho(A)=\sup \{\rho>0: x \in A, B(x ; \rho) \subset A\},
$$

where $B(x ; \rho)$ is the open ball with centre $x$ and radius $\rho$. The following will be used in the proof of Theorem 1

Lemma 3. If $\Omega$ is an open, convex set in $\mathbb{R}^{m}$ with inradius $\rho(\Omega)$ and with finite Lebesgue measure $|\Omega|$ then $\Omega$ is bounded, and

$$
\operatorname{diam}(\Omega) \leq 2 m \omega_{m-1}^{-1} \rho(\Omega)^{1-m}|\Omega| .
$$

If $\Omega$ is an open, convex set in $\mathbb{R}^{m}$ with finite Lebesgue measure $|\Omega|$, then

$$
\rho(\Omega) \geq 2^{m-1}\left(m \omega_{m}\right)^{-1} \operatorname{diam}(\Omega)^{1-m}|\Omega| .
$$

Proof. Let $d$ be any point of the boundary of an open, convex set $\Omega$ with Lebesgue measure $|\Omega|$, and let 0 be the centre of an open ball with radius $\rho(\Omega)$ in $\Omega$. Let $\Pi$ be the $(m-1)$-dimensional plane through 0 perpendicular to the straight line segment $[0, d]$. The $\operatorname{disc} \Pi \cap B(0 ; \rho(\Omega))$ has $(m-1)$-dimensional Lebesgue measure $\omega_{m-1} \rho(\Omega)^{m-1}$. By convexity we have that the cone with base $\Pi \cap B(0 ; \rho(\Omega))$ and vertex $d$ is contained in $\Omega$. Since the Lebesgue measure of that cone is given by $m^{-1} \omega_{m-1}|d| \rho(\Omega)^{m-1}$, we conclude that

$$
|\Omega| \geq m^{-1} \omega_{m-1}|d| \rho(\Omega)^{m-1} .
$$

This implies that $\Omega$ is bounded. Then by (13),

$$
\begin{aligned}
\operatorname{diam}(\Omega) & =\sup \left\{\left|d_{1}-d_{2}\right|: d_{1} \in \partial \Omega, d_{2} \in \partial \Omega\right\} \\
& \leq 2 \sup \{|d|: d \in \partial \Omega\} \\
& \leq 2 m \omega_{m-1}^{-1} \rho(\Omega)^{1-m}|\Omega| .
\end{aligned}
$$

This proves (11).

To prove (12), we have by [16] that

$$
\rho(\Omega) \geq \mathcal{P}(\Omega)^{-1}|\Omega| .
$$

Since $\Omega$ is convex and contained in a ball with radius $\frac{1}{2} \operatorname{diam}(\Omega)$ we have by Proposition 2.4.3 (i) in [3] that

$$
\mathcal{P}(\Omega) \leq m \omega_{m}\left(\frac{1}{2} \operatorname{diam}(\Omega)\right)^{m-1} .
$$

Inequality (12) follows from (14) and (15).

Below we obtain estimates for $|\mathcal{T}(A)-\mathcal{T}(B)|$ and $\left|\lambda_{k}(A)-\lambda_{k}(B)\right|$ for two bounded convex sets $A$ and $B$ in terms of their Hausdorff distance $d^{H}(A, B)$.

Lemma 4. If $A$ and $B$ are two open bounded convex sets in $\mathbb{R}^{m}$, if $\mathcal{T}$ is a set function satisfying hypotheses (a) and (b), and if $\epsilon:=d^{H}(A, B) \leq \rho(A) / 2$ then

$$
|\mathcal{T}(A)-\mathcal{T}(B)| \leq \frac{2 \tau 3^{\tau} \epsilon}{\rho(A)} \mathcal{T}(A),
$$

and

$$
\left|\lambda_{k}(A)-\lambda_{k}(B)\right| \leq \frac{16 \epsilon}{\rho(A)} \lambda_{k}(A) .
$$


Proof. Define the $\epsilon$-neighbourhood of a set by $\Omega^{\epsilon}=\left\{x \in \mathbb{R}^{m}: \operatorname{dist}(x, \Omega)<\epsilon\right\}$. Then $d^{H}(A, B)=\epsilon$ implies $B \subset A^{\epsilon}$ and $A \subset B^{\epsilon}$. But since $A$ is convex $A^{\epsilon} \subset\left(1+\frac{\epsilon}{\rho(A)}\right) A$, where the latter homothety is with respect to the centre of an inball. Then by monotonicity and scaling we have that for $\epsilon \leq \rho(A) / 2$,

$$
\begin{aligned}
\mathcal{T}(B)-\mathcal{T}(A) & \leq \mathcal{T}\left(A^{\epsilon}\right)-\mathcal{T}(A) \\
& \leq \mathcal{T}\left(\left(1+\frac{\epsilon}{\rho(A)}\right) A\right)-\mathcal{T}(A) \\
& =\left(\left(1+\frac{\epsilon}{\rho(A)}\right)^{\tau}-1\right) \mathcal{T}(A) \\
& \leq \frac{\tau 3^{\tau} \epsilon}{2^{\tau} \rho(A)} \mathcal{T}(A) .
\end{aligned}
$$

Reversing the roles of $A$ and $B$ we obtain by using $\rho(B) \geq \rho(A)-\epsilon$, and (18) that for $\epsilon \leq \rho(A) / 2$,

$$
\begin{aligned}
\mathcal{T}(A)-\mathcal{T}(B) & \leq\left(\left(1+\frac{\epsilon}{\rho(B)}\right)^{\tau}-1\right) \mathcal{T}(B) \\
& \leq\left(\left(1+\frac{\epsilon}{\rho(A)-\epsilon}\right)^{\tau}-1\right)(\mathcal{T}(B)-\mathcal{T}(A)+\mathcal{T}(A)) \\
& \leq\left(\left(1+\frac{\epsilon}{\rho(A)-\epsilon}\right)^{\tau}-1\right)\left(1+\frac{\epsilon}{\rho(A)}\right)^{\tau} \mathcal{T}(A) \\
& \leq \frac{2 \tau 3^{\tau} \epsilon}{\rho(A)} \mathcal{T}(A) .
\end{aligned}
$$

Inequality (16) follows by (18) and (19).

To prove (17) we use the scaling and monotonicity of the Dirichlet eigenvalues to obtain that

$$
\begin{aligned}
\lambda_{k}(A)-\lambda_{k}(B) & \leq \lambda_{k}(A)-\lambda_{k}\left(A^{\epsilon}\right) \\
& \leq \lambda_{k}(A)-\lambda_{k}\left(\left(1+\frac{\epsilon}{\rho(A)}\right) A\right) \\
& =\left(1-\left(1+\frac{\epsilon}{\rho(A)}\right)^{-2}\right) \lambda_{k}(A) \\
& \leq \frac{2 \epsilon}{\rho(A)} \lambda_{k}(A) .
\end{aligned}
$$

Reversing the roles of $A$ and $B$ we obtain that

$$
\lambda_{k}(B)-\lambda_{k}(A) \leq \frac{2 \epsilon}{\rho(B)} \lambda_{k}(B) .
$$

Since $A \subset B^{\epsilon} \subset\left(1+\frac{\epsilon}{\rho(B)}\right) B$ we have that $\left(1+\frac{\epsilon}{\rho(B)}\right)^{-1} A \subset B$. Hence

$$
\lambda_{k}(B) \leq\left(1+\frac{\epsilon}{\rho(B)}\right)^{2} \lambda_{k}(A)
$$

So by (21) and (22) we obtain by using $\rho(B) \geq \rho(A)-\epsilon$ and $\epsilon \leq \rho(A) / 2$ that

$$
\begin{aligned}
\lambda_{k}(B)-\lambda_{k}(A) & \leq \frac{2 \epsilon}{\rho(B)}\left(1+\frac{\epsilon}{\rho(B)}\right)^{2} \lambda_{k}(A) \\
& \leq \frac{16 \epsilon}{\rho(A)} \lambda_{k}(A) .
\end{aligned}
$$

Inequality (17) follows from (20) and (23). 
In order to prove Theorem 1 (i) we let $c>0$, fix $k \in \mathbb{N}$, and let $\left(\Omega_{k, n}\right)_{n \in \mathbb{N}}$ be a minimizing sequence of (4). We first show that $\operatorname{diam}\left(\Omega_{k, n}\right)$ is uniformly bounded in $n$. It follows from (5) and hypothesis (b) that for any convex $\Omega$ with finite Lebesgue measure

$$
T^{*} \leq \frac{\mathcal{T}(\Omega)}{|\Omega|^{\tau / m}} .
$$

Since $\mathcal{T}\left(\Omega_{k, n}\right)=c$ we have that

$$
\left|\Omega_{k, n}\right| \leq\left(\frac{c}{T^{*}}\right)^{m / \tau} .
$$

By hypothesis (c1), $T^{*}>0$, and so the left hand side of (25) is uniformly bounded from above in $n$. Hence the spectrum of the Dirichlet Laplacian acting in $L^{2}\left(\Omega_{k, n}\right)$ is discrete. We may assume without loss of generality that for all $n \in \mathbb{N}, \lambda_{k}\left(\Omega_{k, n}\right) \leq 2 I_{k}(c)$. Hence

$$
\lambda_{1}\left(\Omega_{k, n}\right) \leq \lambda_{k}\left(\Omega_{k, n}\right) \leq 2 I_{k}(c) .
$$

It is well-known that for a convex set $\Omega$ in $\mathbb{R}^{m}$ the spectrum of the Dirichlet Laplacian acting in $L^{2}(\Omega)$ is bounded from below by $(2 \rho(\Omega))^{-2}$, see 6 . It follows that $\lambda_{1}\left(\Omega_{k, n}\right) \geq\left(2 \rho\left(\Omega_{k, n}\right)\right)^{-2}$. By (26) we conclude that

$$
\rho\left(\Omega_{k, n}\right) \geq 2^{-3 / 2} I_{k}(c)^{-1 / 2} .
$$

By (25), (27) and (11) we have that $\operatorname{diam}\left(\Omega_{k, n}\right)$ is bounded uniformly in $n$ and satisfies

$$
\operatorname{diam}\left(\Omega_{k, n}\right) \leq \frac{m 2^{(3 m-1) / 2}}{\omega_{m-1}}\left(\frac{c}{T^{*}}\right)^{m / \tau} I_{k}(c)^{(m-1) / 2} .
$$

Hence there exists a sequence of translates of $\left(\Omega_{k, n}\right)$, again denoted by $\left(\Omega_{k, n}\right)$, contained in a sufficiently large closed cube $B_{k}$. Then $\left(\overline{\Omega_{k, n}}\right)_{n \in \mathbb{N}}$ is a sequence of compact sets in $B_{k}$. The collection of compact subsets of $B_{k}$ is compact in the Hausdorff metric by Theorem 2.4.10 in [11. Hence there exists a subsequence, again denoted by $\left(\overline{\Omega_{k, n}}\right)_{n \in \mathbb{N}}$ such that $\left(\overline{\Omega_{k, n}}\right)$ converges in the Hausdorff metric to a compact set say $K_{k}$. Then $K_{k}$ is convex (Section 2.2 in [1]), and by (27),

$$
\rho\left(K_{k}\right) \geq 2^{-3 / 2} I_{k}(c)^{-1 / 2} .
$$

We conclude that the interior of $K_{k}$, denoted by $\Omega_{k}^{*}$, is non-empty. Hence $\Omega_{k, n}$ converges to $\Omega_{k}^{*}$ in the Hausdorff metric. Since $\mathcal{T}\left(\Omega_{k, n}\right)=c, \rho\left(\Omega_{k, n}\right) \geq$ $2^{-3 / 2} I_{k}(c)^{-1 / 2}$ we have by (16) with $A=\Omega_{k, n}$ and $B=\Omega_{k}^{*}$ that $\mathcal{T}\left(\Omega_{k}^{*}\right)=c$. Furthermore

$$
\left|\lambda_{k}\left(\Omega_{k}^{*}\right)-I_{k}(c)\right| \leq\left|\lambda_{k}\left(\Omega_{k, n}\right)-I_{k}(c)\right|+\left|\lambda_{k}\left(\Omega_{k, n}\right)-\lambda_{k}\left(\Omega_{k}^{*}\right)\right| .
$$

The first term in the right hand side of (28) tends to 0 as $n \rightarrow \infty$, since $\left(\Omega_{k, n}\right)$ is a minimizing sequence. To estimate the second term in that right hand side we use (17) with $A=\Omega_{k, n}$ and $B=\Omega_{k}^{*}$. Since $\rho\left(\Omega_{k, n}\right) \geq 2^{-3 / 2} I_{k}(c)^{-1 / 2}$ and $\lambda_{k}\left(\Omega_{k, n}\right) \leq 2 I_{k}(c)$ this term tends to 0 too as $n \rightarrow \infty$. Hence $\lambda_{k}\left(\Omega_{k}^{*}\right)=I_{k}(c)$ and $\Omega_{k}^{*}$ is a minimizer. This proves $1(\mathrm{i})$. 
Since, by (27), the inradius is uniformly bounded from below and since all elements of the minimizing sequence are convex the convergence is also in the complementary Hausdorff metric.

To prove part (ii) of Theorem 1 we consider the set $D$ defined by (6), and choose $\alpha_{c}$ such that $\mathcal{T}\left(\alpha_{c} D\right)=c$. By scaling we have that

$$
\alpha_{c}=\left(\frac{c}{\mathcal{T}(D)}\right)^{1 / \tau}
$$

Hence

$$
I_{k}(c) \leq \lambda_{k}\left(\alpha_{c} D\right)=\alpha_{c}^{-2} \lambda_{k}(D)=\left(\frac{\mathcal{T}(D)}{c}\right)^{2 / \tau} \lambda_{k}(D) .
$$

Furthermore by Corollary 1 in [14], we have that for any open set $\Omega$ in $\mathbb{R}^{m}$ with finite Lebesgue measure,

$$
\lambda_{k}(\Omega) \geq \frac{m C_{m}}{m+2}\left(\frac{k}{|\Omega|}\right)^{2 / m}
$$

where

$$
C_{m}=4 \pi^{2} \omega_{m}^{-2 / m}
$$

It follows by (31) and (30) that

$$
\begin{aligned}
\left|\Omega_{k}^{*}\right| & \geq\left(\frac{m C_{m}}{m+2}\right)^{m / 2} \frac{k}{\lambda_{k}\left(\Omega_{k}^{*}\right)^{m / 2}} \\
& \geq\left(\frac{m C_{m}}{m+2}\right)^{m / 2} \frac{k}{I_{k}(c)^{m / 2}} \\
& \geq\left(\frac{m C_{m}}{m+2}\right)^{m / 2}\left(\frac{c}{\mathcal{T}(D)}\right)^{m / \tau} \frac{k}{\lambda_{k}(D)^{m / 2}} .
\end{aligned}
$$

By Weyl's Theorem (see e.g. Theorem 10.6 in [17]) we have that

$$
\lambda_{k}(D)=C_{m} k^{2 / m}+o\left(k^{2 / m}\right), k \rightarrow \infty .
$$

We conclude by (32) and (33) that

$$
\liminf _{k \rightarrow \infty}\left|\Omega_{k}^{*}\right| \geq\left(\frac{m}{m+2}\right)^{m / 2}\left(\frac{c}{\mathcal{T}(D)}\right)^{m / \tau} .
$$

Hence by (7) and (34) we have that

$$
\limsup _{k \rightarrow \infty} \operatorname{diam}\left(\Omega_{k}^{*}\right) \leq K\left(\frac{m+2}{m}\right)^{(t \tau-1) / 2} c^{1 / \tau} \mathcal{T}(D)^{(t \tau-1) / \tau} .
$$

Hence there exists a sufficiently large cube $B$ which contains translates of $\Omega_{k}^{*}, k=1,2, \cdots$ again denoted by $\Omega_{k}^{*}, k=1,2, \cdots$. As before $\left(\overline{\Omega_{k}^{*}}\right)_{k \in \mathbb{N}}$ is a sequence of compact sets in $B$. The collection of compact subsets of $B$ is compact, in the Hausdorff metric by Theorem 2.4.10 in [11. Hence there exists a 
convergent subsequence $\left(\overline{\Omega_{k_{l}}^{*}}\right)_{l \in \mathbb{N}}$ which converges to a convex, compact set $K$. By (12), (34) and (35) we have that

$$
\begin{aligned}
\rho(K) & =\lim _{l \rightarrow \infty} \rho\left(\Omega_{k_{l}}^{*}\right) \geq \liminf _{k \rightarrow \infty} \rho\left(\Omega_{k}^{*}\right) \\
& \geq 2^{m-1} K^{1-m}\left(m \omega_{m}\right)^{-1}\left(\frac{m}{m+2}\right)^{(t \tau(m-1)+1) / 2} c^{1 / \tau} \mathcal{T}(D)^{(t \tau(1-m)-1) / \tau} .
\end{aligned}
$$

Then the interior of $K$, denoted by $\Omega^{*}$, is non-empty. We now use (16) with $A=\Omega_{k_{l}}^{*}$ and $B=\Omega^{*}$ to conclude that $\mathcal{T}\left(\Omega^{*}\right)=c$. Let $\epsilon>0$ be arbitrary. There exists $l_{0} \in \mathbb{N}$ such that $l \geq l_{0}$ implies $\Omega_{k_{l}}^{*} \subset \Omega^{* \epsilon}$. By monotonicity of Dirichlet eigenvalues $\lambda_{k_{l}}\left(\Omega^{* \epsilon}\right) \leq \lambda_{k_{l}}\left(\Omega_{k_{l}}^{*}\right) \leq \lambda_{k_{l}}\left(\alpha_{c} D\right)$. It follows that, by applying Weyl's Theorem to both sides of the inequality above, $\left|\Omega^{* \epsilon}\right| \geq\left|\alpha_{c} D\right|=\alpha_{c}^{m}$. Since $\epsilon>0$ was arbitrary we conclude that $\left|\Omega^{*}\right| \geq\left|\alpha_{c} D\right|=\alpha_{c}^{m}$. On the other hand, by (24) and (29) we have that

$$
\left|\Omega^{*}\right| \leq\left(\frac{\mathcal{T}\left(\Omega^{*}\right)}{\mathcal{T}(D)}\right)^{m / \tau}=\left(\frac{c}{\mathcal{T}(D)}\right)^{m / \tau}=\alpha_{c}^{m} .
$$

By uniqueness of the set $D$ in (5) the only set which satisfies the constraint in (4) and has measure $\alpha_{c}^{m}$ is an isometry of $\alpha_{c} D$. We conclude that the subsequence $\Omega_{k_{l}}^{*}$ converges in the Hausdorff metric to an isometry of $\alpha_{c} D$. This concludes the proof of (8) since the limit is independent of the subsequence.

The convergence also takes place in the complementary Hausdorff metric since the elements in the sequence are convex, and by (8), their inradii are uniformly bounded from below. This concludes the proof Theorem 1(ii).

Corollary 5. Let $\Omega_{k}^{*}$ be a minimizer of

$$
I_{k}(c)=\inf \left\{\lambda_{k}(\Omega): \Omega \text { open, convex in } \mathbb{R}^{m}, \mathcal{P}(\Omega)=c\right\},
$$

then there exists a sequence of translates of $\left(\Omega_{k}^{*}\right)$, again denoted by $\left(\Omega_{k}^{*}\right)$, which converges to the ball with perimeter $c$ in the Hausdorff metric.

Proof. It follows by the isoperimetric inequality that if $\mathcal{P}(\Omega)=c$ then $|\Omega|<\infty$, and so the Dirichlet Laplacian acting in $L^{2}(\Omega)$ has discrete spectrum. The scaling relation under (b) holds with $\tau=m-1$. By 8 ] we have that the diameter bound under (d) holds with $t=m-1$, and reads

$$
\operatorname{diam}(\Omega) \leq m^{2-m} \omega_{m-1}^{-1} \mathcal{P}(\Omega)^{m-1}|\Omega|^{2-m} .
$$

The unique set $D$ under (6) is the ball in $\mathbb{R}^{m}$ with Lebesgue measure 1 .

Note that the constant in (37) is sharp for a sequence of double sided cones with diameter increasing to infinity 8 .

Recall that the moment of inertia of an open set $\Omega$ in $\mathbb{R}^{m}$ with respect to its centre of mass is defined by

$$
\mathcal{J}(\Omega)=\frac{1}{2|\Omega|} \iint_{\Omega \times \Omega} d x d y|x-y|^{2} .
$$


Corollary 6. Let $\Omega_{k}^{*}$ be a minimizer of

$$
I_{k}(c)=\inf \left\{\lambda_{k}(\Omega): \Omega \text { open, convex in } \mathbb{R}^{m}, \mathcal{J}(\Omega)=c\right\},
$$

Then there exists a sequence of translates of $\left(\Omega_{k}^{*}\right)$, again denoted by $\left(\Omega_{k}^{*}\right)$ which converges in the Hausdorff metric to the ball with moment of inertia $c$.

Proof. From (38) it is clear that the moment of inertia is invariant under isometries and monotone on the open sets. Hence (a) is satisfied. By (38) we see that the scaling under (b) holds with $\tau=m+2$. The isoperimetric inequality for the moment of inertia states that

$$
\mathcal{J}(\Omega) \geq \frac{m}{m+2} \omega_{m}^{-2 / m}|\Omega|^{(m+2) / m},
$$

with equality if and only if $\Omega$ is a ball (up to sets of measure 0 ). The isoperimetric inequality (39) implies that (c2) holds for the ball with Lebesgue measure 1. Below we show that the diameter bound under (d) holds with $t=\frac{1}{2}$, and reads

$$
\operatorname{diam}(\Omega) \leq K \mathcal{J}(\Omega)^{1 / 2}|\Omega|^{-1 / 2},
$$

where

$$
K=4\left(m(m+1)^{2}(m+2)\right)^{1 / 2} .
$$

Note that by (11) an open, convex set with finite Lebesgue measure is bounded. To prove (40) we let $d$ and $f$ be two points of the boundary of $\Omega$ such that $|d-f|=\operatorname{diam}(\Omega)$. Let $p$ be any point of the straight line segment $[d, f]$, and let $\Pi_{p}$ be the $(m-1)$-dimensional plane perpendicular to $[d, f]$. We denote $\Omega_{p}=\Pi_{p} \cap \Omega$, and denote its $(m-1)$-dimensional Lebesgue measure by $\left|\Omega_{p}\right|_{m-1}$. Then $p \mapsto\left|\Omega_{p}\right|_{m-1}$ is continuous on the compact line segment $[d, f]$, and attains its maximum at say 0 . We choose the positive $x_{1}$ axis along $[0, f]$, and put $x=\left(x_{1}, x^{\prime}\right)$. Without loss of generality we may assume that $|f| \geq \frac{1}{2} \operatorname{diam}(\Omega)$. By (38) we have that

$$
\begin{aligned}
\mathcal{J}(\Omega) & \geq \frac{1}{2|\Omega|} \int_{0}^{|f|} d x_{1} \int_{0}^{|f|} d y_{1} \int_{\Omega_{x_{1}}} d x^{\prime} \int_{\Omega_{y_{1}}} d y^{\prime}\left(\left(x_{1}-y_{1}\right)^{2}+\left|x^{\prime}-y^{\prime}\right|^{2}\right) \\
& \geq \frac{1}{2|\Omega|} \int_{0}^{|f|} d x_{1} \int_{0}^{|f|} d y_{1} \int_{\Omega_{x_{1}}} d x^{\prime} \int_{\Omega_{y_{1}}} d y^{\prime}\left(x_{1}-y_{1}\right)^{2} \\
& =\frac{1}{2|\Omega|} \int_{0}^{|f|} d x_{1} \int_{0}^{|f|} d y_{1}\left|\Omega_{x_{1}}\right|_{m-1}\left|\Omega_{y_{1}}\right|_{m-1}\left(x_{1}-y_{1}\right)^{2} .
\end{aligned}
$$

By convexity we have that $\Omega$ contains the cone with base $\Omega_{x_{1}}$ and vertex $f$. It follows by monotonicity and scaling that

$$
\left|\Omega_{x_{1}}\right|_{m-1} \geq\left|\Omega_{0}\right|_{m-1}\left(1-\frac{x_{1}}{|f|}\right)^{m-1} .
$$


By (42) and (43) we find that

$$
\begin{aligned}
\mathcal{J}(\Omega) & \geq \frac{\left|\Omega_{0}\right|_{m-1}^{2}}{2|\Omega|} \int_{0}^{|f|} d x_{1} \int_{0}^{|f|} d y_{1}\left(1-\frac{x_{1}}{|f|}\right)^{m-1}\left(1-\frac{y_{1}}{|f|}\right)^{m-1}\left(x_{1}-y_{1}\right)^{2} \\
& =\frac{\left|\Omega_{0}\right|_{m-1}^{2}|f|^{4}}{2|\Omega|} \int_{0}^{1} d x_{1} \int_{0}^{1} d y_{1}\left(1-x_{1}\right)^{m-1}\left(1-y_{1}\right)^{m-1}\left(x_{1}-y_{1}\right)^{2} \\
& =\frac{\left|\Omega_{0}\right|_{m-1}^{2}|f|^{4}}{m(m+1)^{2}(m+2)|\Omega|} \\
& \geq \frac{\left|\Omega_{0}\right|_{m-1}^{2} \operatorname{diam}(\Omega)^{4}}{16 m(m+1)^{2}(m+2)|\Omega|} \\
& \geq \frac{\operatorname{diam}(\Omega)^{2}|\Omega|}{16 m(m+1)^{2}(m+2)}
\end{aligned}
$$

where we have used that $|\Omega| \leq\left|\Omega_{0}\right|_{m-1} \operatorname{diam}(\Omega)$. This implies (40), (41).

Corollary 7. If $\mathcal{T}$ satisfies (a),(b) and (c1) then

$$
H_{k}=\inf \left\{\lambda_{k}(\Omega)+\mathcal{T}(\Omega): \Omega \text { open, convex in } \mathbb{R}^{m}\right\},
$$

has a minimizer which is up to isometries a homothety of the minimizer of (4) with $c=1$.

The proof of this corollary is straightforward and is deferred to the Appendix.

\section{Proof of Theorem 2}

In this section we will prove Theorem 2

Proof. (i) It is evident from the definition of $J_{k}(c)$ that $c \mapsto J_{k}(c)$ is monotonically decreasing. To prove continuity we let $c_{2}>0$ and we let $\epsilon>0$ be arbitrary. Then there exists an open set $\Omega_{\epsilon}$ such that $\lambda_{k}\left(\Omega_{\epsilon}\right) \leq(1+\epsilon) J_{k}\left(c_{2}\right)$, and which satisfies the constraints $\left|\Omega_{\epsilon}\right| \leq 1$ and $\mathcal{P}\left(\Omega_{\epsilon}\right) \leq c_{2}$. Let $c_{1}<c_{2}$. Then the open set $\left(\frac{c_{1}}{c_{2}}\right)^{1 /(m-1)} \Omega_{\epsilon}$ satisfies

$$
\left|\left(\frac{c_{1}}{c_{2}}\right)^{1 /(m-1)} \Omega_{\epsilon}\right| \leq\left(\frac{c_{1}}{c_{2}}\right)^{m /(m-1)}<1
$$

and

$$
\mathcal{P}\left(\left(\frac{c_{1}}{c_{2}}\right)^{1 /(m-1)} \Omega_{\epsilon}\right) \leq c_{1}
$$

Hence

$$
J_{k}\left(c_{1}\right) \leq \lambda_{k}\left(\left(\frac{c_{1}}{c_{2}}\right)^{1 /(m-1)} \Omega_{\epsilon}\right) \leq(1+\epsilon)\left(\frac{c_{2}}{c_{1}}\right)^{2 /(m-1)} J_{k}\left(c_{2}\right) .
$$

This implies (10) since $\epsilon>0$ was arbitrary. To prove continuity we have for $c_{1}<c_{2}$ by (10) and the monotonicity of $c \mapsto J_{k}(c)$ that

$$
0 \leq J_{k}\left(c_{1}\right)-J_{k}\left(c_{2}\right) \leq J_{k}\left(c_{2}\right)\left(\left(\frac{c_{2}}{c_{1}}\right)^{2 /(m-1)}-1\right) .
$$


This implies left-continuity at $c_{2}$. We have by (10) and monotonicity of $c \mapsto$ $J_{k}(c)$ that

$$
0 \leq J_{k}\left(c_{1}\right)-J_{k}\left(c_{2}\right) \leq J_{k}\left(c_{1}\right)\left(1-\left(\frac{c_{1}}{c_{2}}\right)^{2 /(m-1)}\right) .
$$

This implies right-continuity at $c_{1}$.

(ii) Suppose that $c>\mu_{k}$. By the definition of $\mu_{k}$ there exists $\Omega^{*} \in \mathfrak{M}_{k}$ with $\mathcal{P}\left(\Omega^{*}\right) \leq c$. Hence $J_{k}(c) \leq \lambda_{k}\left(\Omega^{*}\right)=M_{k}(1)$. Since trivially $J_{k}(c) \geq M_{k}(1)$ we have that $J_{k}(c)=M_{k}(1)$ for $c>\mu_{k}$, and that $\Omega^{*}$ is a minimizer of (9). Finally $J_{k}\left(\mu_{k}\right)=M_{k}(1)$ by the continuity of $c \mapsto J_{k}(c)$ proved above.

(iii) Suppose that $c<\pi_{k}^{-(m-1) / m}$. Let $\mathfrak{P}_{k}(c)$ denote the collection of minimizers of $P_{k}(c)$, and put

$$
\pi_{k}(c)=\inf \left\{|\Omega|: \Omega \in \mathfrak{P}_{k}(c)\right\} .
$$

By scaling we have that

$$
\begin{aligned}
\pi_{k}(c) & =\inf \left\{\left|c^{1 /(m-1)} \Omega\right|, c^{1 /(m-1)} \Omega \in \mathfrak{P}_{k}(c)\right\} \\
& =c^{m /(m-1)} \inf \left\{|\Omega|: \Omega \in \mathfrak{P}_{k}(1)\right\} \\
& =c^{m /(m-1)} \pi_{k} .
\end{aligned}
$$

First suppose that $\pi_{k}(c)<1$. Then there exists a minimizer $\tilde{\Omega} \in \mathfrak{P}_{k}(c)$ with $|\tilde{\Omega}| \leq 1$. Hence $J_{k}(c) \leq \lambda_{k}(\tilde{\Omega}) \leq P_{k}(c)$. On the other hand, $J_{k}(c) \geq P_{k}(c)$. It follows that $J_{k}(c)=P_{k}(c), \Omega_{*}:=c^{-1 /(m-1)} \tilde{\Omega} \in \mathfrak{P}_{k}$, and that $c^{1 /(m-1)} \Omega_{*}$ is a minimizer of (9). We have that $J_{k}(c)=P_{k}(c)$ if $\pi_{k}(c)=1$ by the continuity of $c \mapsto J_{k}(c)$ proved above.

(iv) Let $\Omega^{*} \in \mathfrak{M}_{k}$. Then $\left|\Omega^{*}\right|=1$, and $\mathcal{P}\left(\Omega^{*}\right) \geq m \omega_{m}^{1 / m}$ by the isoperimetric inequality.

(v) Let $\Omega_{*} \in \mathfrak{P}_{k}$. Then $\mathcal{P}(\Omega)=1$, and $|\Omega| \leq m^{-m /(m-1)} \omega_{m}^{-1 /(m-1)}$ by the isoperimetric inequality.

(vi) By the Li-Yau inequality (31),

$$
P_{k}(1)=\lambda_{k}\left(\Omega^{*}\right) \geq \frac{m C_{m}}{m+2}\left(\frac{k}{\left|\Omega^{*}\right|}\right)^{2 / m} .
$$

For the cube $Q_{a} \in \mathbb{R}^{m}$ with $\left|Q_{a}\right|=a^{m}$ and with $\mathcal{P}\left(Q_{a}\right)=1$ we have that

$$
a=(2 m)^{-1 /(m-1)} \text {. }
$$

We have that $\lambda_{k}\left(Q_{a}\right) \geq \lambda_{1}\left(Q_{a}\right)=m \pi^{2} / a^{2}$. Since for $x>1$ we have that $\max \{n \in \mathbb{N}: n<x\}=\lfloor x\rfloor \geq x / 2$, we conclude that

$$
\begin{aligned}
k & =\sharp\left\{\left(k_{1}, \cdots, k_{m}\right) \in \mathbb{N}^{m}: \pi^{2}\left(k_{1}^{2}+\cdots+k_{m}^{2}\right) \leq \lambda_{k}\left(Q_{a}\right) a^{2}\right\} \\
& \geq\left(\sharp\left\{k \in \mathbb{N}: m \pi^{2} k^{2}<\lambda_{k}\left(Q_{a}\right) a^{2}\right\}\right)^{m} \\
& \geq\left\lfloor\frac{a \lambda_{k}\left(Q_{a}\right)^{1 / 2}}{\pi m^{1 / 2}}\right]^{m} \\
& \geq\left(\frac{a \lambda_{k}\left(Q_{a}\right)^{1 / 2}}{2 \pi m^{1 / 2}}\right)^{m} .
\end{aligned}
$$

It follows that

$$
\lambda_{k}\left(Q_{a}\right) \leq \frac{4 \pi^{2} m}{a^{2}} k^{2 / m} .
$$


We also have that $P_{k}(1) \leq \lambda_{k}\left(Q_{a}\right)$. Putting this together with (45), (46) and (47) we conclude that

$$
\left|\Omega^{*}\right| \geq(2 m)^{-m /(m-1)}(m+2)^{-m / 2} \omega_{m}^{-1} .
$$

\section{Appendix}

In this appendix we prove the following.

Proposition 8. Let

$$
L_{k}=\inf \left\{\lambda_{k}(\Omega): \Omega \text { open in } \mathbb{R}^{m}, \mathcal{T}(\Omega) \leq 1\right\},
$$

and

$$
T_{k}=\inf \left\{\lambda_{k}(\Omega)+\mathcal{T}(\Omega): \Omega \text { open in } \mathbb{R}^{m}\right\} .
$$

Suppose $\mathcal{T}$ satisfies the hypotheses (a), (b) and (c1). Then the variational problem defined under (48) has a minimizer if and only if the variational problem under (49) has a minimizer. Moreover these minimizers are hometheties of one another.

Proof. It is convenient to define

$$
N_{k}=\inf \left\{\lambda_{k}(\Omega) \mathcal{T}(\Omega)^{2 / \tau}: \Omega \text { open in } \mathbb{R}^{m}, \mathcal{T}(\Omega)<\infty\right\} .
$$

First we show that $L_{k}=N_{k}$. We have by scaling of the Dirichlet eigenvalues that

$$
\begin{aligned}
L_{k} & =\inf \left\{\lambda_{k}(t \Omega): \Omega \text { open in } \mathbb{R}^{m}, t>0, \mathcal{T}(t \Omega) \leq 1\right\} \\
& =\inf \left\{t^{-2} \lambda_{k}(\Omega): \Omega \text { open in } \mathbb{R}^{m}, t>0, t^{\tau} \mathcal{T}(\Omega) \leq 1\right\} \\
& \geq \inf \left\{\mathcal{T}(\Omega)^{2 / \tau} \lambda_{k}(\Omega): \Omega \text { open in } \mathbb{R}^{m}, t>0, t^{\tau} \mathcal{T}(\Omega) \leq 1\right\} \\
& \geq \inf \left\{\mathcal{T}(\Omega)^{2 / \tau} \lambda_{k}(\Omega): \Omega \text { open in } \mathbb{R}^{m}, \mathcal{T}(\Omega)<\infty\right\} \\
& =N_{k} .
\end{aligned}
$$

We obtain the reverse inequality by choosing $t=\mathcal{T}(\Omega)^{-1 / \tau}$ in the second line of (51). If $\Omega^{*}$ is a minimizer of (48) then $\mathcal{T}\left(\Omega^{*}\right)=1$. Hence $\mathcal{T}\left(\Omega^{*}\right)^{2 / \tau} \lambda_{k}\left(\Omega^{*}\right)=$ $\lambda_{k}\left(\Omega^{*}\right)=L_{k}=N_{k}$, and the infimum in (50) is attained for $\Omega^{*}$. Conversely if $\Omega^{*}$ is a minimizer of $(50)$ then we choose $\alpha>0$ such that $\mathcal{T}\left(\alpha \Omega^{*}\right)=1$. Hence $\alpha=\mathcal{T}\left(\Omega^{*}\right)^{-1 / \tau}$. So $\alpha \Omega^{*}$ satisfies the constraint in (48), and $\lambda_{k}\left(\alpha \Omega^{*}\right)=$ $\alpha^{-2} \lambda_{k}\left(\Omega^{*}\right)=\mathcal{T}\left(\Omega^{*}\right)^{2 / \tau} \lambda_{k}\left(\Omega^{*}\right)=N_{k}=L_{k}$. Hence the infimum in (48) is attained by a homothety of $\Omega^{*}$. We conclude that (48) has a minimizer if and only if (50) has a minimizer.

Next we show that the variational problem under (49) has a minimizer if and only if the variational problem under (50) has a minimizer. We note that

$$
\begin{aligned}
T_{k} & =\inf \left\{\lambda_{k}(\Omega)+\mathcal{T}(\Omega): \Omega \text { open in } \mathbb{R}^{m}\right\} \\
& =\inf \left\{\lambda_{k}(t \Omega)+\mathcal{T}(t \Omega): \Omega \text { open in } \mathbb{R}^{m}, t>0\right\} \\
& =\inf \left\{t^{-2} \lambda_{k}(\Omega)+t^{\tau} \mathcal{T}(\Omega): \Omega \text { open in } \mathbb{R}^{m}, t>0, \mathcal{T}(\Omega)<\infty\right\}
\end{aligned}
$$


The infimum over $t>0$ is attained for

$$
t(\Omega)=\left(\frac{2 \lambda_{k}(\Omega)}{\tau \mathcal{T}(\Omega)}\right)^{1 /(\tau+2)} .
$$

Hence

$$
T_{k}=n(\tau) \inf \left\{\lambda_{k}(\Omega)^{\tau /(\tau+2)} \mathcal{T}(\Omega)^{2 /(\tau+2)}: \Omega \text { open in } \mathbb{R}^{m}\right\}=n(\tau) N_{k}^{\tau /(\tau+2)},
$$

where

$$
n(\tau)=\left(\frac{\tau}{2}\right)^{2 /(\tau+2)}+\left(\frac{2}{\tau}\right)^{\tau /(\tau+2)} .
$$

If $\Omega^{*}$ is a minimizer of (50) then by (49), (52) and (53),

$$
\begin{aligned}
T_{k} & \leq \lambda_{k}\left(t\left(\Omega^{*}\right) \Omega^{*}\right)+\mathcal{T}\left(t\left(\Omega^{*}\right) \Omega^{*}\right) \\
& =t\left(\Omega^{*}\right)^{-2} \lambda_{k}\left(\Omega^{*}\right)+t\left(\Omega^{*}\right)^{\tau} \mathcal{T}\left(\Omega^{*}\right) \\
& =n(\tau) N_{k}^{\tau /(\tau+2)} \\
& =T_{k} .
\end{aligned}
$$

Hence $t\left(\Omega^{*}\right) \Omega^{*}$ is a minimizer of (49). If $\Omega^{*}$ is a minimizer of (49) then by (53) we have that

$$
\begin{aligned}
N_{k}^{\tau /(\tau+2)} & \leq\left(\lambda_{k}\left(\Omega^{*}\right) \mathcal{T}\left(\Omega^{*}\right)^{2 / \tau}\right)^{\tau /(\tau+2)} \\
& =n(\tau)^{-1} \inf \left\{t^{-2} \lambda_{k}\left(\Omega^{*}\right)+t^{\tau} \mathcal{T}\left(\Omega^{*}\right): t>0\right\} \\
& =n(\tau)^{-1} \inf \left\{\lambda_{k}\left(t \Omega^{*}\right)+\mathcal{T}\left(t \Omega^{*}\right): t>0\right\} \\
& \leq n(\tau)^{-1}\left(\lambda_{k}\left(\Omega^{*}\right)+\mathcal{T}\left(\Omega^{*}\right)\right) \\
& =n(\tau)^{-1} T_{k} \\
& =N_{k}^{\tau /(\tau+2)}
\end{aligned}
$$

Hence $\Omega^{*}$ is a minimizer of (50). We conclude that (49) has a minimizer if and only if (50) has a minimizer. This also concludes the proof of the proposition.

Proof of Corollary 7 . We remark that Proposition 8 holds if the variational expressions under (48) and (49) have an additional convexity constraint. If $\mathcal{T}$ satisfies (a), (b) and (c1) then (4) has a minimizer. By the previous remark we have that Proposition 8 implies that the variational expression under (44) has a minimizer which is a homothety of the one corresponding to (4).

\section{References}

[1] M. van Den Berg, M. Iversen, On the minimization of Dirichlet eigenvalues of the Laplace operator, J. Geometric Analysis 23, 660-676 (2013).

[2] D. Bucur, Minimization of the $k$-th eigenvalue of the Dirichlet Laplacian, Arch. Ration. Mech. Anal. 206, 1073-1083 (2012). 
[3] D. Bucur, G. Buttazzo, Variational methods in shape optimization problems, Progress in nonlinear differential equations and their applications, Birkhäuser Verlag, Boston (2005).

[4] D. Bucur, G. Buttazzo, A. Henrot, Minimization of $\lambda_{2}(\Omega)$ with a Perimeter Constraint, Indiana University Mathematics Journal 58, 2709 2728 (2009).

[5] D. Bucur, P. Freitas, Asymptotic behaviour of optimal spectral planar domains with fixed perimeter, J. Mathematical Physics 54, 053504 (2013).

[6] E. B. DAvies, A Review of Hardy inequalities, The Maz'ya anniversary Collection 2, Oper. Theory Adv. Appl. 110, 55-67 (1999).

[7] G. De Philippis, B. VelichKov, Existence and regularity of minimizers for some spectral functionals with perimeter constraint, Appl. Math. Optim. 69, 199-231 (2014).

[8] P. Gritzmann, J. Wills, D. Wrase, A new isoperimetric inequality, J. Reine Angew. Math. 379, 22-30 (1987).

[9] A. Henrot, Extremum Problems for Eigenvalues of Elliptic Operators, Frontiers in Mathematics, Birkhäuser Verlag, Basel (2006).

[10] A. Henrot, E. Oudet, Minimizing the second eigenvalue of the Laplace operator with Dirichlet boundary conditions, Arch. Ration. Mech. Anal. 169, 73-87 (2003).

[11] A. Henrot, M. Pierre, Variation et optimisation de formes: Une analyse géométrique, Mathématiques et Applications 48, Springer, Berlin (2005).

[12] M.-T. KohLER-JoBin, Démonstration de l'inégalité isopérimétrique $P \lambda^{2} \geq \pi j_{0}^{4} / 2$, conjecture par Pólya et Szegö, C. R. Acad. Sci. Paris Sr. A-B 281, A119-A121 (1975).

[13] M.-T. KohleR-Jobin, Une méthode de comparaison isopérimétrique de fonctionnelles de domaines de la physique mathématique. I. Une démonstration de la conjecture isopérimétrique $P \lambda^{2} \geq \pi j_{0}^{4} / 2$ de Pólya et Szegö, Z. Angew.Math. Phys. 29, 757-766 (1978).

[14] P. LI, S. T. YAU, On the Schrödinger equation and the eigenvalue problem, Comm. Math. Phys. 88, 309-318 (1983).

[15] D. Mazzoleni, A. Pratelli, Existence of minimizers for spectral problems, J. Math. Pures Appl. 100, 433-453 (2013).

[16] R. Osserman, Bonnesen-style isoperimetric inequalities, Amer. Math. Monthly 86, 1-29 (1979).

[17] B. Simon, Functional Integration and Quantum Physics, Academic Press, New York, NY, 1979.

[18] S. A. Wolf, J. B. Keller, Range of the First Two Eigenvalues of the Laplacian, Proc. R. Soc. Lond. A 447, 397-412 (1994). 